\title{
A Jumping Genes Scheme for Multi-objective Coordinated Voltage Control
}

\author{
H.M. Ma \\ Electronic Engineering Department, \\ City University of Hong Kong \\ Kowloon \\ Hong Kong \\ Haomin.ma@student.cityu.edu.hk
}

\author{
K.F.Man \\ Electronic Engineering Department, \\ City University of Hong Kong \\ Kowloon \\ Hong Kong \\ eekman@cityu.edu.hk
}

\author{
David J.Hill \\ Research School of Info Sciences and \\ Engineering, \\ The Australian National University \\ Canberra \\ ACT,0200, Australia \\ David.hill@anu.edu.au
}

\begin{abstract}
A}$ new jumping genes evolutionary algorithm is proposed for searching the agreeable solution for retaining the voltage profile. On the basis of optimal coordinated voltage control structure, this scheme was testified using the New England 39-bus power system. The main feature of this optimization method is its ability to find the extreme non-dominated solutions that conld fulfill the voltage control requirement even when the objective function are contradicted with each other.
\end{abstract}

Index Terms-evolutionary algorithm, jumping genes, coordinated voltage control, multi-objective optimization, Pareto front

\section{INTRODUCTION}

Apart from transient instability, voltage instability always shows a medium or long term behavioural effect in losing the normal profile of voltages and sometime even, developing into a catastrophic voltage collapse. There has been increasing interest in the study of voltage instability problems in recent years.

In modern power networks, generators are usually large and far away from major load centres. The major transmission lines are typically long. This situation, generally, can be rectified using two basic approaches to control the system voltages [1], (i) adjust the voltage at the generating plant by the use of excitation controllers, (ii) adjust voltage control devices close to load. For the former case, this method is not effective for improving the voltage at the load end [1], whereas the later one the controllers, capacitors, load tap changers, and load shedding installed in the load centre should be considered to maintain the voltage profile.

Coordinated voltage control (CVC) is a control scheme for recovering voltage instability. It is not only focused on the facility used based on local information and single device but also coordinated with power system from other areas [2]. In this way, a better quality of voltage control is ensured by several actions at various locations rather than the use of a single control action.

With the development of computer and communication technology, the model-based predictive control is now a well established process control technique that can be applied in power systems [2-3]. With the full modelled power system and its current states, the short term system output trajectory of a predictive horizon can be calculated according to candidate control sequences. A cost function of minimizing the difference between the predictive trajectory and the reference output is adopted to gain the optimal control performance. This kind of control methodology is generally referred to as model predictive control (MPC).

Optimal coordinated voltage control (OCVC) of a modern power system constitutes a multi-objective measures that are designated to govern the voltage profile. For a real world power system, there are some other objectives should be fulfilled such as the customer requirement and load shedding avoidance. Load shedding is activated only if the constraint cannot be satisfied using any combination of other normal controls, such as LTC and capacitors [2]. In this paper, the minimization of the load shedding is considered as one of the prime objective functions.

A newly developed jumping genes evolutionary algorithm (JGEA) [9] that is incorporated into the non-dominated sorting genetic algorithm (NSGA) [8-9] is proposed to optimize the voltage control problem in this paper. JGEA consists only a simple operation in which a transposition of the gene(s) is allowed to jump either within the same chromosome or to other chromosome in the population pool while the framework of Genetic Algorithm [7,8] remains unchanged.

The non-dominated solution sets searched by JGEA provide a landscape of control for coordinated voltage control. Researchers and operators may choose their desirable solutions for voltage emergency according to different preferences.

The organization of this paper is as follows: section 2 is to describe the power system and its control issues. The multi-objective issue for governing the OCVC is introduced in section 3 . The simulation and comparison results are listed in section 4 . Finally, some conclusions are given in section 5 .

\section{MODELING AND CONTROL PROBLEM}

\subsection{System Modelling}

The power system models can be expressed in the hybrid differential-algebraic (DA) form (1) [2].

$$
\begin{aligned}
& \dot{x}=f(t, x, w, z(k)) \\
& 0=g(x, w, z(k))
\end{aligned}
$$

Where, $x$ are dynamic state variables that its value can't change instantaneously, $w$ are algebraic state variables that can change instantaneously due to changes in $x$ or $z(k) . z(k)$ denotes discrete control variables that can change only at fixed time instants given at a selected sample time. 
For voltage stability, the algebraic state variables are voltages at buses which can be changed instantaneously. The discrete control variables are those secondary voltage control devices, like tap-changers, switching capacitors and so on, which are controlled at a fixed time. In coordinated voltage control problem, an optimized scheme should be developed for scheduling the use of those voltage control devices.

The aggregate exponential recover (AER) load model by Hill and Karlsson has been widely used in the research of voltage control. This model has been verified in several field tests and parameters for a wide range of operating conditions. We considered all bus loads as this AER model (2) [5].

$$
\begin{aligned}
& \dot{x}_{i, p}=-\frac{1}{T_{i, p}} x_{i, p}+P_{i, 0}\left(V_{i}^{\alpha_{s}}-V_{i}^{\alpha_{i}}\right), \\
& P_{d i}=\left(1-n_{d i} D_{s h e d}\right)\left(\frac{x_{i, p}}{T_{i, p}}+P_{i, 0} V_{i}^{\alpha_{t}}\right), \\
& \dot{x}_{i, q}=-\frac{1}{T_{i, q}} x_{i, q}+Q_{i, 0}\left(V_{i}^{\beta_{s}}-V_{i}^{\beta_{s}}\right), \\
& Q_{d i}=\left(1-n_{d i} D_{\text {shed }}\right)\left(\frac{x_{i, q}}{T_{i, q}}+Q_{i, 0} V_{i}^{\beta_{t}}\right),
\end{aligned}
$$

Where, $T_{i, p}$ and $T_{i q}$ are the time constants; $P_{d i}$ and $Q_{d i}$ are the active power and the reactive power, respectively; $D_{\text {shed }}$ is the load shedding step size, $n_{d i}$ is the number of the load shedding steps, $x_{i, p}$ and $x_{i, q}$ are the internal load state, $\alpha$ and $\beta$ are load index parameters.

\subsection{New England 39-bus power system}

The one-line diagram of New England 39-bus power system is used for this study. It is shown in Fig.1. 12 on load tap change transformers and 19 load shedding can be used as voltage control devices.

The ratios of load tap changers can be changed in steps of 0.02 between 0.80 p.u. to 1.20 p.u.. The load tap changers are blocked if the primary side voltage falls below 0.93p.u. for $10 \mathrm{~s}$. The total load that can be shed for each bus is up to $50 \%$ of its initial value in steps of $5 \%$. The load shedding can be applied when the associated bus voltage drops below 0.90 p.u. for $5 \mathrm{~s}$.

A fault happed at $15 \mathrm{~s}$ as the Generator 32 is tripped. The system voltage would collapse if no control is used. The voltage responses at buses 11,12 and 13 are illustrated in the Fig.2.

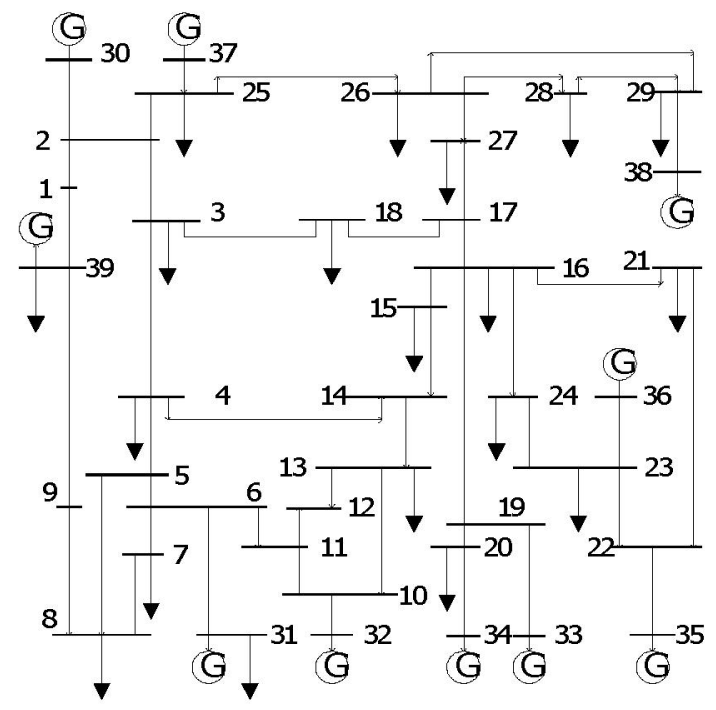

Fig.1 The New England ten-generator-39-bus power system

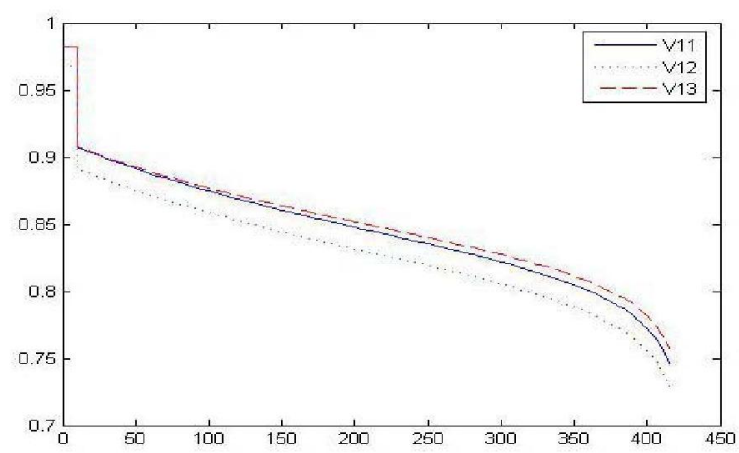

Fig. 2 Voltages of bus 11, bus 12 and bus 13, without control

The numerical simulation of full DA model, (1), over the prediction horizon is the most accurate method of power system simulation. But it is time consuming. The Euler state prediction (ESP) brought in [2] is more efficient and can capture the long-term behavior of voltage instability. The long-term voltage behavior is typically monotonic during voltage collapse scenarios. Based on this assumption, the output trajectories are approximated by straight lines between their respective values at the beginning and end of the prediction interval.

\subsection{MPC based CVC}

CVC is a scheme relies upon the simulated performance of a power system in accordance to the predefined system model that governs the devices connection, control device's statues, and current states of the power system. On the basis of performance, a series of control strategies can be determined for enhancing the overall voltages of the power system. 
It includes three aspects of system design: 1) sequencing: to decide the order of control actions in a discrete sequence domain with an unknown number of stages; 2) timing: to decide the switching time of each control action in a real number domain; 3) tuning: to decide the values of the adjustable parameters of each control action when it is activated [4].

As mentioned above, CVC in this paper is based on the model predictive control strategy. The principle of MPC contains the following three ideas: a) explicit use of a model to predict the progress output along a future time horizon; $b$ ) calculation of a control sequence to optimize a performance index, and c) a receding horizon strategy, so that at each instant the horizon is moved along to the future, which involves the application of the first control signal of the sequence calculated at each step [8].

\section{MULTI-OBJECTIVE OPTIMIZATION}

\subsection{Non-dominated solutions of multi-objective problems}

The presence of multiple objectives optimization, in principle, gives rise to a set of optimal solutions (largely known as Pareto-optimal solutions) other than a single globally optimized solution. In the absence of any further information, one of these obtained Pareto-optimal solutions cannot be said to be better than the other [6] but each may fulfill the design requirement. Given that with $n$ objectives for consideration, the process of minimization is thus:

$$
\operatorname{Min} f_{1}(x), f_{2}(x), \cdots, f_{n}(x)
$$

where $f_{1}(\cdot), f_{2}(\cdot), \ldots, f_{n}(\cdot)$ are $\mathrm{n}$ objectives to be minimized.

When following inequalities hold between two solutions $\mathrm{x}$ and $\mathrm{y}$, the solution $\mathrm{y}$ is said to dominate the solution $\mathrm{x}$ :

$$
f_{i}(y) \leq f_{i}(x) \text { for } \forall i \text { and } \exists j: f_{j}(y)<f_{j}(x)
$$

If a solution is not dominated by any other solutions, that solution is said to be a non-dominated solution. The set of all possible non-dominated solutions constitutes a non-dominated front, Fig. 3 .

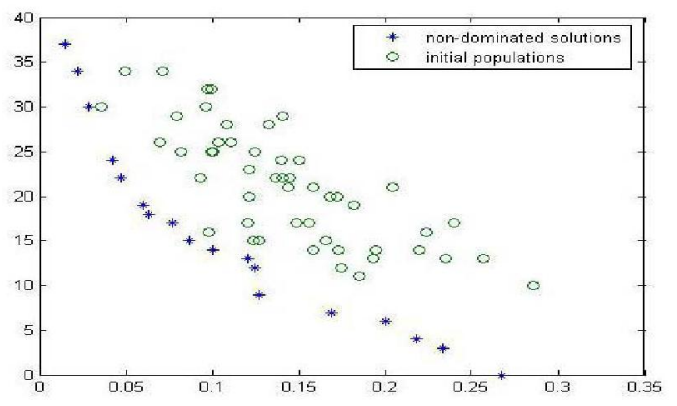

Fig.3 Non-dominated solutions
This front may not be the true Pareto-optimal front as this front may or may not be existed for a real world system. Therefore, it's best to be considered as close as to the reference front [11]

In the case of optimizing the power system for voltage stability, the most important control targets are to keep bus voltages at the expect level. So the objective function can be defined as,

$$
J_{v}=\min \sum_{i}\left|V_{i}-V_{r e f}\right|
$$

where $V_{i}$ denotes the final voltage at bus $i ; V_{\text {ref }}$ denotes the reference voltage at bus $i$.

At the same time, the requirement of customs is also the essential criterion to be fulfilled by the power system. In our study, we model the issue of load shedding as the other objective to be minimized

$$
J_{l}=\min \sum n_{\text {loadi }}
$$

where $n_{\text {loadi }}$ denotes the shedding steps of load shedding on bus $i$.

\subsection{Non-dominated sorting genetic algorithms (NSGA)}

In NSGA [6,7], the generation of parents is sorted with its' offspring according to the rank of each chromosomes. The rank of a chromosome is decided by the number of chromosomes who dominates. So the group of solutions which have the same rank as 1 is called the non-dominated solutions.

The step of "non-dominated sorting" is to divide all populations into different groups according to their ranks. All non-dominated solutions of the population are assigned in rank-I. Then the individuals with rank equals to 2 are grouped to rank-II. The procedure of classification is finished when all individuals are grouped.

The step of "Selection" is different with that of standard GAs for those ranked groups of non-dominated solutions. We reference the fast NSGA of NSGA-II in [7]. The priority of selection is according to the rank of each solution. So the group of solutions which have the rank-I will be selected first. If it does not reach the population number $\mathrm{N}$, the group of rank-II will be selected. If $\mathrm{N}$ is still not reached, the selection will move to the next group. While there are solutions of $2 \mathrm{~N}$ are selected, the selection will stop when $\mathrm{N}$ chromosomes for the next turn of crossover and mutation is obtained.

The step of "Non-dominated sorting" and "Selection" is shown in Fig.4. 


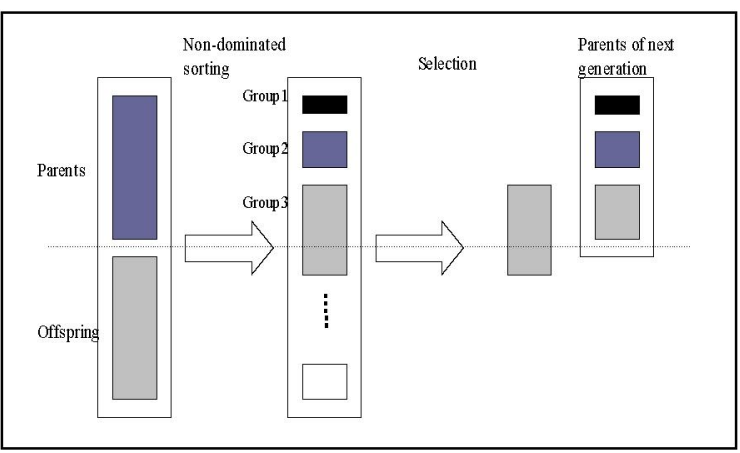

Fig. 4 "Non-dominated sorting" and "Selection"

\subsection{Jumping gene evolutionary algorithms (JGEA)}

JGEA [9] is an improved algorithm shows high ability in finding multiobjective solutions particularly at those extreme ends of the Pareto front. JGGA emulates the phenomenon of corn plants experiment by Barbara McClintock [10].

It was noticed that one of the chromosomes tended to break from generation to generation. The two parts of this chromosome would dissociate from each other, called "Dissociation" (Ds). The Ds can change its position in the chromosome means that the genes can move or "Transpose" around the genome. Then the gene is said can "jump". Jumping genes can move around the genome by cut and paste, which means a piece of DNA is cut and pasted in somewhere. Also, they can move by copy and paste. That is the genes remain at the same location while the message in the DNA is copied into RNA and them copied back into DNA at another place in the genome.

The beauty of JGEA is its simplicity in genetic operation and yet provides vastly dimensional variations of building blocks. A transposition of the gene(s) can be induced within the same chromosome or to mate allied chromosomes within the framework of genetic algorithm. It is a more effective strategy than those random mutations. The reasons for this are: (1) JGEA increases the probability of recombination; and (2) higher probability of destroying the harmful connection of a genome which will have less surviving progeny.

\subsection{Encoding and optimization}

The control devices could begin to work at $30 \mathrm{~s}$. The actions of tap-changers may take the form as (i) minus one step and (ii) add one step. The load shedding can be shed for $50 \%$ of its initial value in steps of $5 \%$.

This can be translated into the binary format in the chromosome, such that every two bits expresses the action of a tap-changer and four bits express a load shedding. Assuming there are $\mathrm{m}$ LTCs and $\mathrm{n}$ Load shedding can be used in the coming interval, the structure of chromosome is as in Table.I.
TABLE I

STRUCTURE OF CHROMOSOME

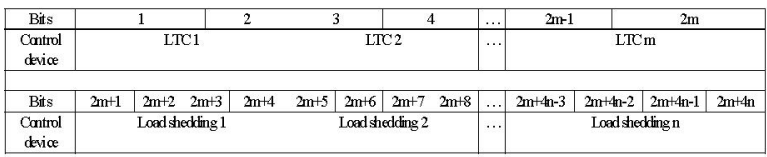

The overall steps of this strategy are as follows:

1. At each control instant $t$, the constrains and limitations of control devices are checked. The structure of chromosomes is set according to the number of active control devices and their control values;

2. Randomly generate the initial population (POP) as candidate control sequences;

3. Calculation the predicted future outputs $\hat{y}_{i}(t+n T), i=1,2, \cdots P O P$, where $n$ is the number of predict horizons, according to the current output of the system $\hat{y}(t)$ and the candidate control which is express by chromosomes. The prediction interval $\left[t, t+t_{p i}\right]$ is expressed by multiple of sample time, $[t, t+n T]$;

4. The predicted outputs are evaluated by the objective functions. In this study, minimize the error between bus voltages and their desired value and minimize the load shedding are the two objective functions;

5. Non-dominated sorting;

6. Ordinary GA operators: selection, crossover and mutation;

7. Jumping genes;

8. Repeat step3-7, until achieving the maximum generation;

9. Get the non-dominated solutions of the finial generation. The best solution is the one which have the minimum value of load shedding among the non-dominated solutions;

10. Controls of the first predict horizon of the best control sequence is used on the system for time $t$ to $t+T$; and

11. Go to step1 for next prediction interval.

\section{SIMULATION RESULTS}

In the example New England power system, the fault happens at 15 second when the Generator tripled on bus 32 . The system control begins at 30 second. That is the first control instant is at $30 \mathrm{~s}$. And the simulations have been carried out with a same sample time and prediction interval of $30 \mathrm{~s}$. There are 12 on load transformers and 19 load shedding. The dynamic load model of (2) is used for the loading on load buses, with $\alpha_{s}=\beta_{s}=0, \alpha_{t}=\beta_{t}=2, T_{p}=T_{q}=60 \mathrm{~s}$.

By using JGEA for searching the solution for control, the system voltages recover to their initial value after 250 seconds. Fig.5 shows the control results of the New England 39-bus power system. These actions are listed in Table.II. Load shedding was naturally avoided in this case for the non-dominated solution of $J_{l}=0$ at each time interval. 


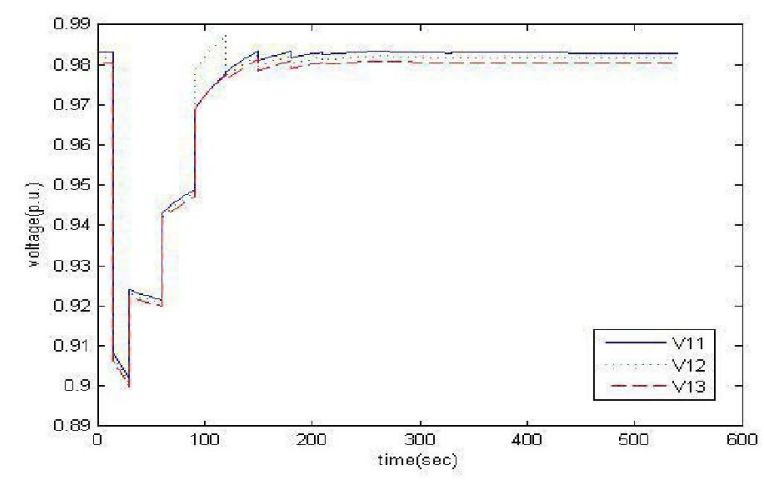

Fig.5 System simulation

The searching results of interval 1 to interval 4 are shown in Fig.6. The indications of $\mathrm{p} 1$ to $\mathrm{p} 4$ are the best controls for each prediction interval. They are all located on the $\mathrm{x}$-axis. (i.e. the objective of $J_{l}$ are zero for these point.) For interval 1 , the system voltage is improved when load shedding increases. For interval 2, interval 3 and interval 4, all load shedding are blocked to their original value and only transformers are used to improve system voltages. We can find that:

TABLE II

CONTROL ACTIONS

\begin{tabular}{|c|c|c|}
\hline Intervals & $\begin{array}{l}\text { Time } \\
(\mathrm{sec})\end{array}$ & Control actions \\
\hline 1 & 30 & $\begin{array}{l}\mathrm{Nt} 31=1.090, \mathrm{Nt} 32=1.090, \mathrm{Nt} 33=1.090, \\
\mathrm{Nt} 35=1.045, \mathrm{Nt36}=1.020, \mathrm{Nt37}=1.045, \\
\mathrm{Nt} 30=1.045, \mathrm{Nt} 38=1.045, \mathrm{Nt} 20=1.080,\end{array}$ \\
\hline 2 & 60 & $\begin{array}{l}\mathrm{Nt} 31=1.110, \mathrm{Nt} 32=1.070, \mathrm{Nt} 33=1.110, \\
\mathrm{Nt} 35=1.065, \mathrm{Nt} 36=1.040, \mathrm{Nt} 37=1.065, \\
\mathrm{Nt} 30=1.065, \mathrm{Nt} 38=1.065, \mathrm{Nt} 20=1.100,\end{array}$ \\
\hline 3 & 90 & $\begin{array}{l}\mathrm{Nt} 11=1.026, \mathrm{Nt} 31=1.130, \mathrm{Nt} 33=1.130, \\
\mathrm{Nt} 35=1.085, \mathrm{Nt} 36=1.060, \mathrm{Nt3}=1.085, \\
\mathrm{Nt} 30=1.085, \mathrm{Nt} 38=1.085, \mathrm{Nt} 20=1.120,\end{array}$ \\
\hline 4 & 120 & $\begin{array}{l}\mathrm{Nt} 11=1.006, \mathrm{Nt} 35=1.065, \mathrm{Nt} 37=1.065, \\
\mathrm{Nt} 30=1.105, \mathrm{Nt} 20=1.140,\end{array}$ \\
\hline 5 & 150 & $\begin{array}{l}\mathrm{Nt} 32=1.090, \mathrm{Nt} 35=1.045, \mathrm{Nt} 36=1.040, \\
\mathrm{Nt} 20=1.160,\end{array}$ \\
\hline 6 & 180 & $\begin{array}{l}\mathrm{Nt} 32=1.110, \mathrm{Nt} 33=1.110, \mathrm{Nt} 35=1.065, \\
\mathrm{Nt} 36=1.060, \mathrm{Nt} 30=1.085, \mathrm{Nt} 20=1.140,\end{array}$ \\
\hline 7 & 210 & $\begin{array}{l}\mathrm{Nt} 32=1.130, \mathrm{Nt} 35=1.085, \mathrm{Nt} 30=1.065, \\
\mathrm{Nt} 38=1.065, \mathrm{Nt} 20=1.160,\end{array}$ \\
\hline 8 & 240 & \\
\hline 9 & 270 & $\begin{array}{l}\mathrm{Nt} 32=1.110, \mathrm{Nt} 33=1.090, \mathrm{Nt} 35=1.105, \\
\mathrm{Nt} 38=1.045, \mathrm{Nt} 20=1.180,\end{array}$ \\
\hline 10 & 300 & \\
\hline 11 & 330 & $\mathrm{Nt} 33=1.070, \mathrm{Nt} 35=1.125$ \\
\hline 12 & 360 & \\
\hline 13 & 390 & \\
\hline 14 & 420 & \\
\hline 15 & 450 & \\
\hline 16 & 480 & \\
\hline 17 & 510 & \\
\hline
\end{tabular}

1) The involved objective functions were found to be compromising with each other. While the load shedding is reduced, the objective function for recover voltages will increase. On the other hand, when load shedding increases, the objective function of recover voltages reduced greatly.

2) On the set of non-dominated solutions, there is one point on the X-axis. That is the location when load-shedding is zero. So load shedding is not necessarily for recovering voltage in this instance. We choose the best control solution from non-dominated front by sorting the number of load shedding. Only the minimum value of load shedding is chosen. This strategy guaranties the objective function of minimum load shedding easily.

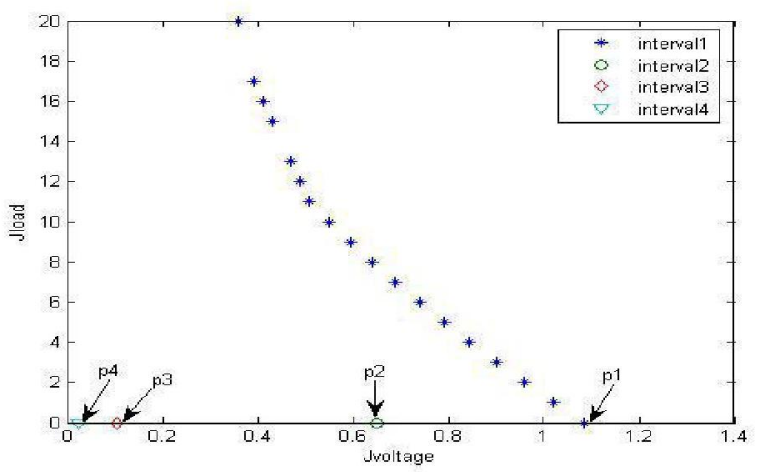

Fig.6 non-dominated solutions of interval 1 to interval 4

\section{CONCLUSION}

The use of proposed JGEA algorithm for searching the optimal control solutions for coordinated voltage control problems based on model predictive control strategy is presented. The voltage profile and the minimization of load shedding are used to govern the performance required. This scheme of control was testified on the basis of the benchmark example of the New England power system. The result indicated that the system voltages could recover to its original voltage successfully by the proposed method. The solution sets found by JGEA can provide a selecting scheme in choosing the appropriate control devices for voltage control.

\section{REFERENCES}

[1] K.T. VU, C.C. Liu, C.W. Taylor, K.M. Jimma, "Voltage Instability: Mechanisms and Control Strategies", Proceedings of The IEEE, Vol.83, No.11, Nov.1995, pp:1442-1355

[2] M. Larsson, D.J. Hill, G. Olsson, "Emergency Voltage Control Using Search and Predictive Control", International Journal of Power. and Energy Systems, Vol.24, No.2, 2002, pp:121-130

[3] J.Y. Wen, Q.H. Wu, D.R. Turner, S.J. Cheng, J. Fitch, "Optimal Coordinated Voltage Control for Power System Voltage Stability", IEEE Transactions on Power Systems, Vol.19, No.2, May 2004, pp:1115-1122

[4] Y.J. Li, D.J. Hill, "Optimal coordinated voltage control of power systems-an immune algorithm solution", 
Control conference, 2004, 5th Asian, Vol.3, July 2004, pp:1398-1403

[5] D.J. Hill, "Nonlinear Dynamic Load Models with Recovery for Voltage Stability Studies", IEEE Transactions on Power Systems, Vol.8, No.1, February 1993, pp:166-176

[6] N. Srinivas, K. Deb, "Multiobjective function optimization using nondominated sorting genetic algorithms", Evolutionary Computation., Vol.2, No.3, 1995, pp:221-248

[7] K. Deb, A. Pratap, S. Agarwal, T. Meyarivan, "A Fast and Elitist Multiobjective Genetic Algorithm: NSGA-II", IEEE Transactions on Evolutionary Computation, Vol.6, No.2, April 2002, pp:182-197

[8] P.D. Roberts, "A Brief Overview of Model Predictive Control", in IEE Seminar on Practical experiences with predictive control, $16 \mathrm{Feb} .2000,2000 / 023$, pp:1-3

[9] K.F. Man, T.M. Chan, K.S. Tang, and S. Kwong, "Jumping Genes in Evolutionary Computing", IEEE IECON2004, Intelligent Control Systems, 2-8 November, Busan, Korea, pp:1268-1272

[10] N. Fedoroff, D. Botstein, The Dynamic Genome, Cold Spring Harbor Laboratory Press, 1992.

[11] P. Czyzak, A. Jaszkiewicz, "Pareto-simulated annealing - a meta-heuristic technique for multi-objective combinatorial optimization", Journal of Multi-Criteria Decision Analysis, Vol.7, 1998, pp:34-47 